significance in the diagnosis or treatment of the disorder, which should be carried out in the usual way, i.e. a common feature of the psychiatric illness, with AIDS concern as a symptom, is hypochondriasis.

However, to avoid missing the obvious, one must not exclude consideration of the possibility of genuine cause for concern, as instanced by two recent male patients who were pathologically preoccupied and terrified that they might have picked up the disease. The first was a basically anxious somewhat hypochondriacal individual aged 60 years, who acknowledged past passive homosexual activity, and the second, a known manic-depressive aged 45 years, confessed his involvement with a prostitute abroad early in 1987.

The first patient required ECT, but the second fortunately responded to conservative antidepressant therapy. Their affective disorders appeared to have heightened awareness of their past sexual contacts and possible consequences, rather than having caused the depression, which was not relieved by pre-test counselling and demonstration (fortunately) that they were HIV sero-negative.

Fenton (Journal, November 1987, 151, 579-588) noted: "Individuals, not only those belonging to the high-risk groups, and known in some cases to be sero-positive but in others not, have developed a terror of and intense preoccupation with AIDS leading to multiple somatic complaints with a conviction of suffering from the disease". Perhaps, then, rather than the terms 'AIDS phobia', 'AIDS panic' or 'pseudo-AIDS', more apposite descriptive and diagnostically acceptable terms might be 'AIDS-concern' or 'AIDS-anxiety'.

Finally, from experience as a consultant AIDS counsellor, if psychiatry does indeed become a 'front-line' speciality in the management of AIDS victims, then provision for staff education (and allocation of financial resources) must be undertaken promptly, not least to minimise the number of 'secondary' cases of this AIDS-related condition among the caretakers.

Halifax General Hospital

M. Segal

Halifax

West Yorkshire

SIR: I refer to the letter from Riccio \& Thompson (Journal, December 1987, 151, 863) commenting on the earlier report by Miller et al (Journal, May 1985, 146, 550-551). I wish to endorse their and others' views, particularly O'Brien's statement (Journal, July $1987,151,127)$ that "What is important in patients presenting with excessive concern about AIDS, but without the disease, is not AIDS itself, but the underlying psychiatric state".

On reading the original report by Miller et al, I expressed my views (Journal, August 1985, 147, 210) making this very point, adding: "Surely we need no further confusion in our already confusing and loose nosology. Do we call a depressive illness characterised in part by either hypochondriacal, overvalued or frankly delusional ideas of cancer (even if the patient has been recently in contact with a cancer victim) a 'pseudo-cancer' syndrome?" I included comments on the article by Miller et al that "What they actually describe, however, are two manifestations of psychiatric disturbance characterised in part by a fear of AIDS resulting in significant impairment but, contrary to the title of the article, they do not convincingly describe "the psychiatric symptoms resulting from a fear of AIDS", which they wish to refer to as 'pseudo-AIDS'. Both these patients were at high risk of contracting AIDS, and further I fear that the invention of a 'pseudo-AIDS syndrome', set against the backcloth of the difficulty of diagnosing AIDS itself in the early stages, might prejudice the diagnosis of AIDS where it actually exists".

It behoves us psychiatrists to be extremely prudent in our use of words, especially so when words might become labels, as labels not infrequently assume the quality of an entity. The history of psychiatry is replete with examples of how words have not clarified issues for us and our patients but have added to the problems which already existed!

Grovelands Priory Hospital

BRON LIPKIN

The Bourne, Southgate

London N14 6RA

\section{Self-Inflicted Eye Injuries}

SIR: The article by Rogers \& Pullen (Journal, November 1987, 151, 691-693) was of much interest as a psychiatric curiosity. Essays such as this, emphasising descriptive psychiatry, are a welcome relief from the usual stuff these days, much of it on epidemiology, surveys, questionnaires, and reports on patients with heavy reliance on complaints and symptoms, i.e. subjective phenomena generated by and reported by patients. It seems to me that a complaint is different from a symptom. In descriptive psychiatry we need to emphasise objective evolution based on signs.

Symptoms and signs often seem to be confused, and may be lumped together. 'Symptom' is from the Greek (semeion) and 'sign' from the Latin (signum). Stedman's dictionary defines semiology (semeiology) as symptomatology, which may be not strictly 